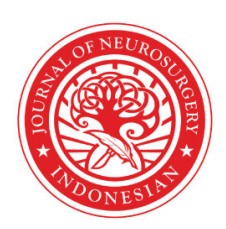

\title{
Acute spontaneous epidural hematoma of cervical spine presenting as quadriparesis in a young patient: a case report
}

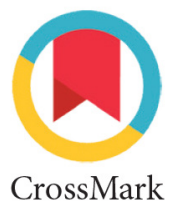

\author{
Uday Kiran Katari ${ }^{1 *}$, Govindappagari Venkateswara Prasanna
}

\section{ABSTRACT}

Introduction: Spontaneous spinal epidural hematoma (SSEH) is a relatively uncommon pathology of the spine. The incidence of SSEH is approximately $0.1-1$ per 100,000 individuals. SSEH can cause acute spinal cord compression and rapid onset of neurologic deficits. Although rare, SSEH must be considered in the differential diagnosis of patients presenting with sudden onset of neurologic symptoms without a history of trauma.

Case presentation: We report a case of a 19-year-old female patient who presented with sudden onset of weakness of all four limbs with pain in the upper cervical region. She has no history of trauma. Magnetic Resonance Imaging (MRI) of the spine was done, which showed an epidural mass in the cervical region, causing severe cord compression. Emergency cervical laminectomy and evacuation of epidural hematoma has was done with excellent post-operative recovery.

Conclusion: Spontaneous spinal epidural hematoma is a relatively rare entity of the spine. The signs and symptoms of this rare condition are non-specific. In patients presenting with a sudden onset of neurologic deficits, the condition should be one of the differential diagnoses. MRI is the investigation of choice in patients suspected to have SSEH. Early surgical intervention results in a better outcome. The level of preoperative neurologic deficit, the severity of the condition, and operative interval are important factors affecting the postoperative outcome.

Keywords: cervical spine, laminectomy, spontaneous spinal epidural hematoma

Cite This Article: Katari, U.K., Prasanna, G.V. 2020. Acute spontaneous epidural hematoma of cervical spine presenting as quadriparesis in a young patient: a case report. Indonesian Journal of Neurosurgery 3(1): 4-7. D0I: 10.15562/ijn.v3i1.65

${ }^{1}$ Consultant Neurosurgeon, Department of Neurosurgery, Simhapuri Institute of Neurological Sciences, Simhapuri Hospital, Nellore, Andhra Pradesh, India

*Corresponding author: Uday Kiran Katari; Department of Neurosurgery, Simhapuri Institute of Neurological Sciences, Simhapuri Hospital, Nellore, Andhra Pradesh, India; uday.krn72@gmail.com

Received: 2019-05-16 Accepted: 2019-09-26 Published: 2020-04-01

\section{INTRODUCTION}

Spontaneous spinal epidural hematoma (SSEH) is a relatively uncommon pathology of the spine. The incidence of SSEH is approximately 0.1 - 1 per 100,000 individuals. ${ }^{1}$ The condition is idiopathic in $40-50 \%$ of the patients. SSEH may be associated with coagulation disorders, vascular malformations, minor injuries to the vertebral column, anti-coagulants and neoplasm. Other predisposing factors include conditions that increase intrathoracic and intraabdominal pressures such as coughing, sneezing, and Valsalva maneuver. $^{2}$

SSEH usually presents with sudden onset of symptoms, which varies from an acute onset of severe radiating neck or back pain that progress towards paraparesis or quadriparesis depending on the level of lesion. ${ }^{3}$ Patients presenting late may develop complete motor and sensory deficits below the level of injury. Neurological status at the time of presentation has prognostic significance as patients with some residual function tend to show complete recovery. ${ }^{4}$

Magnetic Resonance Imaging (MRI) is the diagnostic modality of choice in suspected cases of
SSEH. Acute SSEH presenting within the first 24 hours of the onset of symptoms appears isointense to the cord on T1-weighted (T1W1) images and hyperintense and heterogeneous on T2 weighted (T2W1) images. After 48 hours, the hematoma appears hyperintense on both T1W1 \& T2W1 sequences. ${ }^{5}$ The differential diagnosis includes epidural abscess and spinal epidural lymphoma. ${ }^{6}$

In this study, we report a case of acute SSEH in a young female without any predisposing factors. She presented with acute onset of quadriparesis and neck pain. Emergency cervical laminectomy and evacuation of epidural haematoma was done with excellent neurological outcome.

\section{CASE PRESENTATION}

A 19-year-old female patient presented to the emergency department with complaints of sudden onset weakness of all four limbs and pain in the upper cervical region. There is no history of trauma or activities of physical exertion. She is not using any anticoagulants, and there is no history of bleeding diathesis. Neurologic examination showed upper limb power of grade $2 / 5$, lower limb power of grade 1/5. The tone of all four limbs had decreased. Deep 


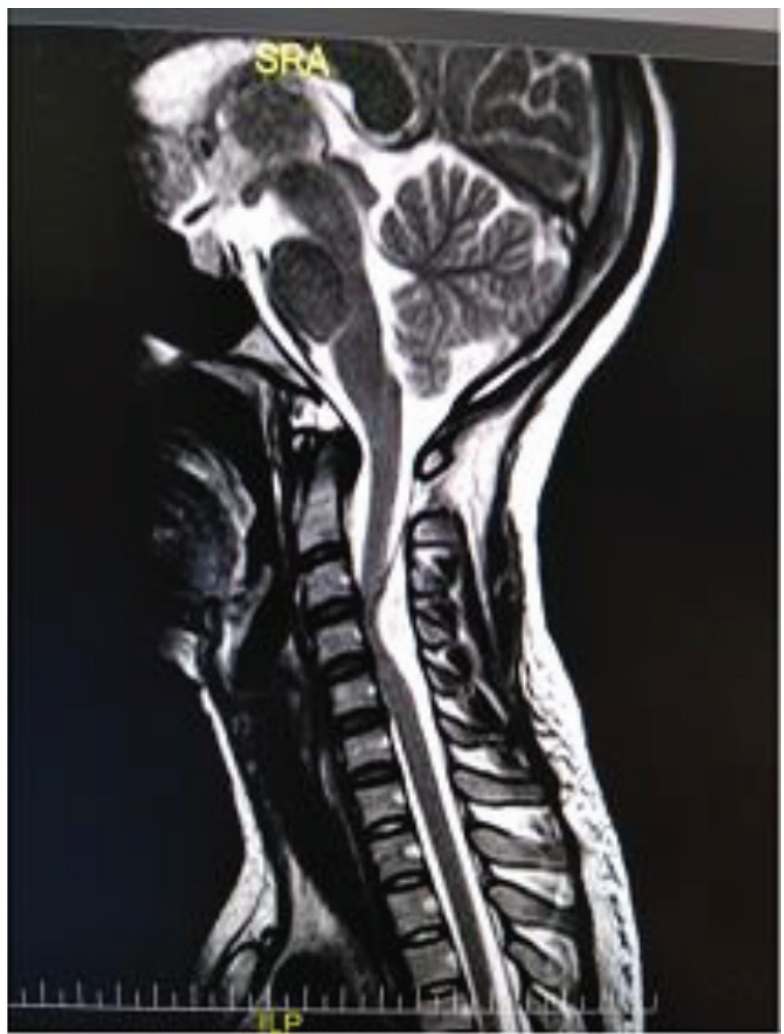

Figure 1. Sagittal T2-weighted magnetic resonance imaging showed a hyperintense lesion at the C3-C5 level, causing compression on the cord.

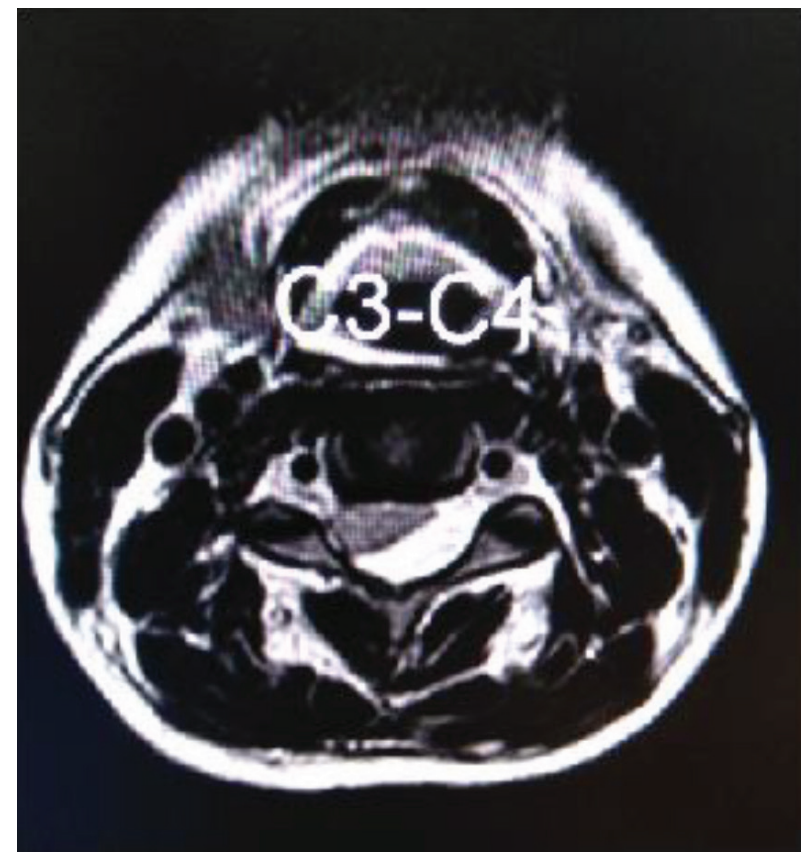

Figure 2. Axial T2-weighted magnetic resonance imaging showed a hyperintense lesion at the $\mathrm{C} 3-\mathrm{C} 4$ level, causing compression on the cord dorsolaterally on the left side. tendon reflexes were absent. Urinary incontinence was present. Cranial nerve examination showed no abnormalities. Decreased sensations to pinprick, light touch and vibratory sense were noted from $\mathrm{C} 4$ dermatome.

An emergency MRI of the spine was done, which showed a well defined extradural lesion from C2 to C6 level measuring $1 \times 1.8 \times 4.0 \mathrm{~cm}$. The lesion was isointense to the cord on T1W1 and hyperintense on T2W1, causing compression on the spinal cord (Figures 1, 2 and 3). Coagulation profile of the patient was normal. The patient was taken up for emergency surgery. The patient underwent C3 laminectomy and evacuation of epidural hematoma (Figure 4).

\section{DISCUSSION}

A spinal epidural hematoma is a rare but serious neurological condition. Spinal epidural hematomas are considered a neurosurgical emergency. SSEH accounts for less than $1 \%$ of all spinal epidural lesions, with an incidence of 1 per million per year. ${ }^{7}$

The condition is idiopathic in $40-50 \%$ of the patients. SSEH may be associated with coagulation disorders, vascular malformations, minor injuries to the vertebral column, anti-coagulants and neoplasm. Other predisposing factors include the conditions which increase intrathoracic and intraabdominal pressures such as coughing, sneezing, and Valsalva maneuver. ${ }^{2}$

However, the exact bleeding source and mechanism of hematoma formation are not known. The age group most frequently affected is $40-50$ years old. The male and female ratio is $1.4: 1{ }^{8}$

Most of the SSEH arise from rupture of the epidural venous plexus. There are two proposed sources of bleeding in cases of SSEH, which are arterial and venous sources. The arterial source of bleeding arises from the "free" anastomotic arteries in the epidural space that connect to radicular arteries. ${ }^{9}$ The venous source of SSEH occurs due to local pooling of blood within valve-less, thinwalled epidural veins and sudden increase in the intrathoracic and intraabdominal pressure elevations leads to epidural vein rupture. Venous bleeding as a source of SSEDH is the commonly accepted hypothesis. ${ }^{10}$

The patient's signs and symptoms depend on the location of epidural hematoma and degree of spinal cord compression. The usual clinical presentation of SSEH is the sudden onset of neck or back pain that progresses towards paraparesis or quadriparesis depending on the level of the lesion and the nerve root. ${ }^{11}$ In the high cervical region, SSEH may cause spinal shock and sudden cardiac arrest. ${ }^{12}$

SSEH is difficult to diagnose based on 


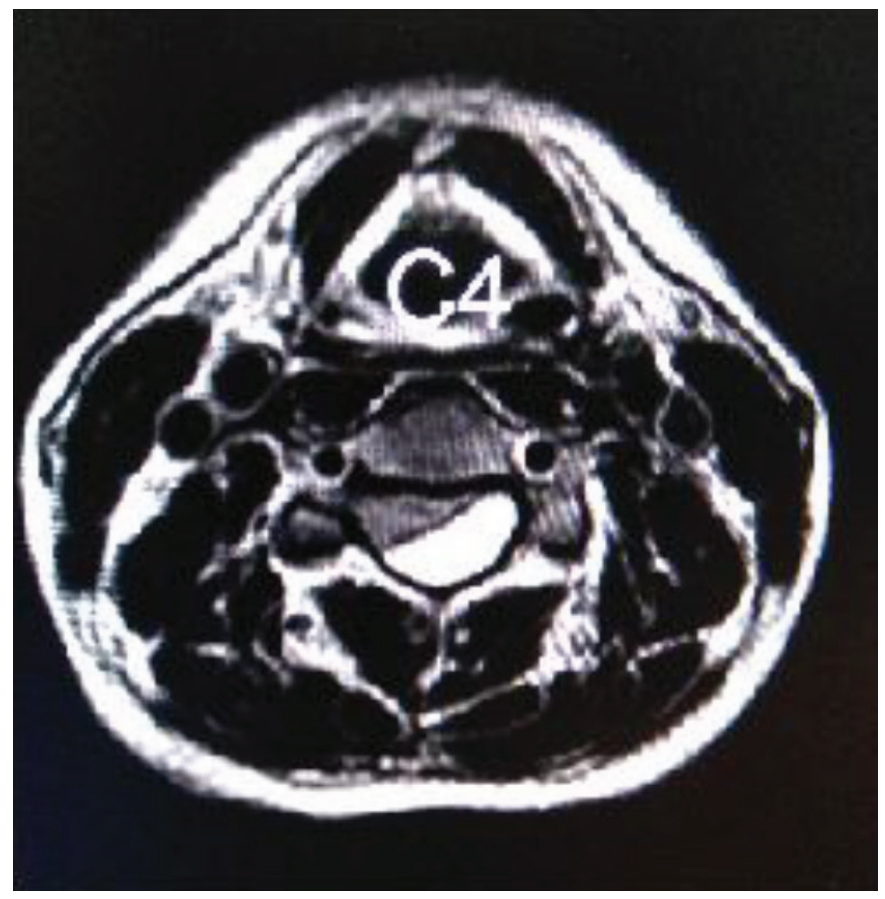

Figure 3. Axial T2-weighted magnetic resonance imaging showed a hyperintense lesion at $\mathrm{C} 4$ level, causing compression on the cord dorsolaterally on the left side.

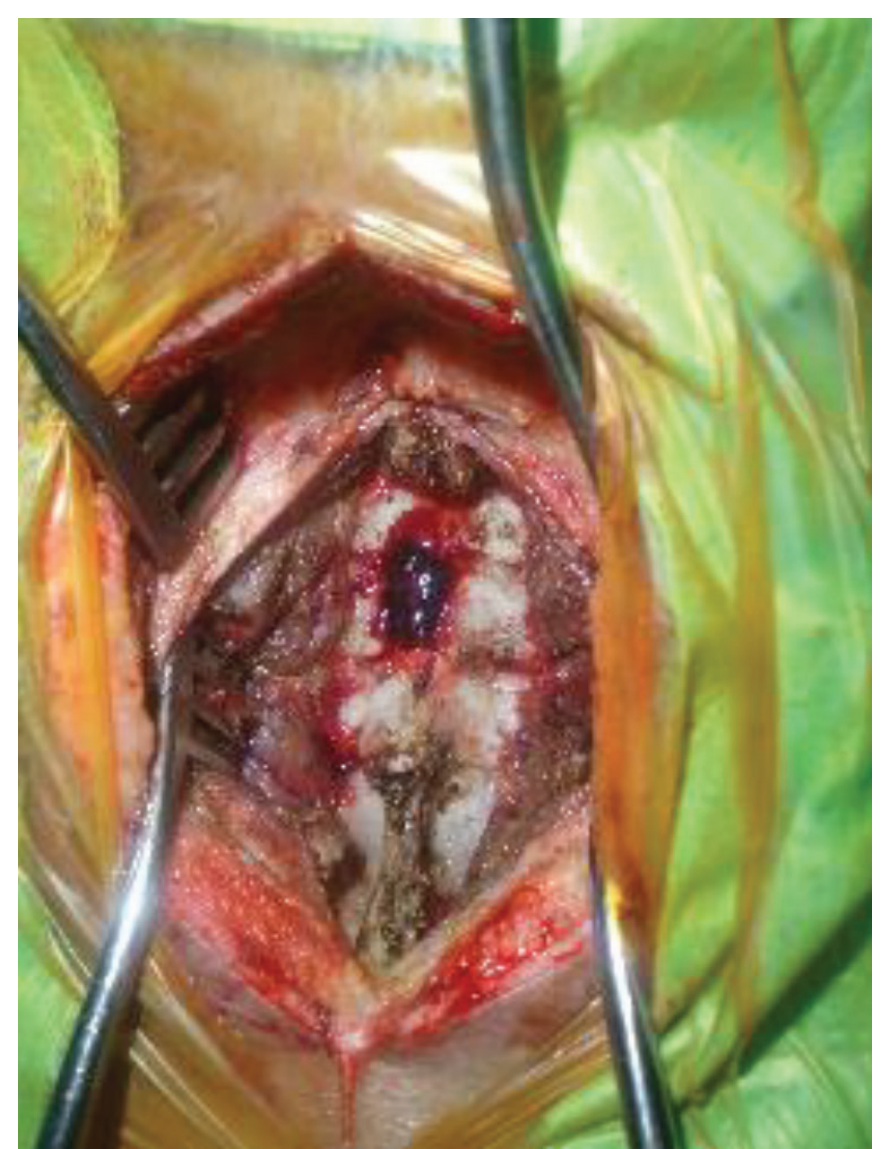

Figure 4. Intraoperative photograph of the patient showing acute spinal epidural hematoma at C3 vertebral level (C3 laminectomy has been done). neurological signs and symptoms. The neurological differential diagnosis includes transverse myelitis, Guillain-Barre Syndrome (GBS), epidural abscess, epidural Subarachnoid Hemorrhage (SAH), acute spinal cord ischemia, epidural tumor, and acute herniated Intervertebral Disc (IVD). ${ }^{13}$

The degree of sensory and motor deficit at the initial presentation determines the prognosis. A patient with incomplete neurologic deficits tends to have good recovery compared to those presenting with complete loss of function. ${ }^{14}$

MRI of the spine shows a characteristic biconvex shaped hematoma in the epidural space with welldefined borders mass effect degree of spinal cord compression. Acute SSEH presenting within the first 24 hours of the onset of symptoms appears isointense to the cord on T1-weighted (T1W1) images and is hyperintense and heterogeneous on T2 weighted (T2W1) images. After 48 hours, the hematoma appears hyperintense on both T1W1 \& T2W1 sequences. ${ }^{5}$ The radiological differential diagnosis includes epidural abscess and spinal epidural lymphoma. ${ }^{6}$

Surgery is the treatment of choice for SSEH, causing compression and mass effect of the spinal cord. Decompressive laminectomy followed by hematoma evacuation was performed to the patient. The patient showed minimal neurologic symptoms, signs of spontaneous neurological recovery and resolving hematoma with no mass effect on MRI and could be managed conservatively. Conservative treatment consists of close observation with a serial of neurologic examinations and MRI imaging. If a patient shows any signs of neurologic deterioration or develops new-onset neurologic symptoms, emergency surgical intervention has to be done.

The factors affecting prognosis in patients with SSEH are the degree of neurologic deficits at the initial presentation and the time interval between presentation and surgery. Patients operated within $12-48$ hours of the onset of symptoms are reported to have shown good neurological recovery. The present patient was operated within 4 hours of the onset of symptoms, and her recovery was satisfactory.

\section{CONCLUSION}

Spontaneous spinal epidural hematoma is a relatively rare entity of spine. The signs and symptoms of this rare condition are non-specific. In patients presenting with sudden onset of neurologic deficits, the condition should be one of the differential diagnoses. MRI is the investigation of choice in patients suspected to have SSEH. Early surgical intervention results in a better outcome. The level of preoperative neurologic deficit, the severity of 
the condition, and operative interval are important factors affecting the postoperative outcome.

\section{CONFLICT OF INTEREST}

There is no potential conflict of interest relevant to this article reported.

\section{PATIENT CONSENT}

Patient consent was obtained from the patient.

\section{FUNDING}

No specific funding was provided for this article.

\section{AUTHORS' CONTRIBUTIONS}

Authors' contributions include manuscript preparation, literature search, manuscript editing and final manuscript.

\section{REFERENCES:}

1. Baek BS, Hur JW, Kwon KY, Lee HK. Spontaneous spinal epidural hematoma. J Korean Neurosurg Soc. 2008; 44(1): 40 - 42. DOI: 10.3340/jkns.2008.44.1.40.

2. Bhat KJ, Kapoor S, Watali YZ, Sharma JR. Spontaneous epidural hematoma of spine associated with clopidogrel: A case study and review of the literature. Asian J Neurosurg. 2015; 10(1): 54. DOI: 10.4103/1793-5482.151521.

3. Groen R, Ponssen H. The spontaneous spinal epidural hematoma. A study of the etiology. J Neurol Sci. 1990; 98(23): 121 - 138. DOI: 10.1016/0022-510x(90)90253-j.

4. Lan T, Chen Y, Yang X-J, Hu S-Y, Guo W-Z, Ren K, et al. Spontaneous spinal epidural haematoma. J Ortho Trans. 2015; 3(3): 152 - 156. DOI: 10.1016/j.jot.2015.03.001.

5. Vazquez-Barquero A, Abascal F, Garcia-Valtuille R, Pinto JI, Figols FJ, Cerezal L. Chronic nontraumatic spinal epidural hematoma of the lumbar spine: MRI diagnosis. Eur Radiol. 2000; 10(10): 1602 - 1605. DOI: 10.1007/s003300000357.

6. Gala FB, Aswani Y. Imaging in spinal posterior epidural space lesions: a pictorial essay. Indian J Radiol Imaging. 2016; 26(3): 299 - 315. DOI: 10.4103/0971-3026.190406.

7. Bakker NA, Veeger NJ, Vergeer RA, Groen RJ. Prognosis after spinal cord and cauda compression in spontaneous spinal epidural hematomas. Neurology. 2015; 84(18): 1894 - 903. DOI: 10.1212/WNL.0000000000001545.

8. Guzel A, Simsek O, Karasalihoglu S, Kucukugurluoglu Y, Acunas B, Tosun A, Cakir B. Spontaneous spinal epidural hematoma after seizure: a case report. Clin Pediatr (Phila). 2007; 46(3): 263 - 265. DOI: 10.1177/0009922806289427.

9. Beatty RM, Winston KR. Spontaneous cervical epidural hematoma. A consideration of etiology. J Neurosurg. 1984; 61(1): 143 - 148. DOI: 10.3171/jns.1984.61.1.0143.

10. Bruyn G. Spinal extradural hematoma. In: Handbook of Clinical Neurology. Amsterdam; North-Holland Publishing Co.; 1976.

11. Yu HP, Fan SW, Yang HL, Tang TS, Zhou F, Zhao X. Early diagnosis and treatment of acute or subacute spinal epidural hematoma. Chin Med J (Engl). 2007; 120(15): 1303 - 1308. DOI: 10.1097/00029330-200708010-00002.

12. Chan DT, Boet R, Poon WS, Yap F, Chan YL. Spinal shock in spontaneous cervical spinal epidural haematoma. Acta Neurochir (Wien). 2004; 146(10): 1161 - 1162; discussion 1162 - 3. DOI: $10.1007 / \mathrm{s} 00701-004-0347-8$.

13. Wang P, Xin X-T, Lan H, Chen C, Liu B. Spontaneous cervical epidural hematoma during pregnancy: case report and literature review. Eur Spine J. 2011; 20(Suppl 2): 176 9. DOI: $10.1007 /$ s00586-010-1484-9.

14. Gopalkrishnan CV, Dhakoji A, Nair S. Spontaneous cervical epidural hematoma of idiopathic etiology: case report and review of literature. J Spinal Cord Med. 2012; 35(2): 113 - 117. DOI: 10.1179/2045772312Y.0000000001.

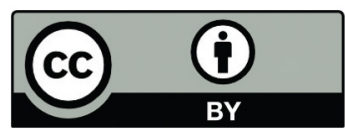

This work is licensed under a Creative Commons Attribution 